九州大学学術情報リポジトリ

Kyushu University Institutional Repository

\title{
THE EFFECT OF SWITCH-OVER DEVICE ON AVAILABILITY OF A 2-DISSIMILAR-UNIT REDUNDANT SYSTEM WITH A WARM STANDBY
}

Kodama, Masanori

Department of Applied Physics, Osaka University

Itoi, Takashi

Department of Applied Physics, 0saka University

https://doi.org/10.5109/13112

出版情報：統計数理研究. 17 (3/4)，pp.39-47，1977-03. Research Association of Statistical Sciences

バージョン :

権利関係 : 


\title{
THE EFFECT OF SWITCH-OVER DEVICE ON AVAILABILITY OF A 2-DISSIMILAR-UNIT REDUNDANT SYSTEM WITH A WARM STANDBY
}

\author{
By \\ Masanori KODAMA* and Takashi ITOI*
}

(Received April 3, 1976)

\begin{abstract}
The effect of switch-over device on reliability of a 2-dissimilarunit redundant system with a warm standby is considered. The Laplace transform of the availability function and steady state availability are derived. The Laplace transform of the reliability function and the mean time to system failure are directly obtained from the Laplace transform of the availability function by suitable transformations. Therefore, a separate analysis of the reliability of the system is redundant. The steady state availability of the parallel and standby system with imperfect switchover are compared.
\end{abstract}

\section{Introduction}

Recently Srinivasan and Gopalan [4] carried out a probabilistic analysis of warm standby system consisting of two similar units and obtained the availability function as well as the reliability function of the system through the method of regeneration points. In their model, they assumed that the system consists of two identical units and switch-over device is perfect. And also they analyzed independently the availability and reliability by setting different equations respectively.

In this paper we consider a warm redundant system consisting of two dissimilar units assuming that the switch-over device is imperfect. We obtain the Laplace tramsform of the availability of the system by supplementary variable technique. The Laplace transform of the reliability function is directly obtained from the availability function by suitable transformations. Explicit expressions for the steady state availability of the system and the mean time to system failure are obtained. The steady state availability of the parallel and standyby system are compared. The optimal system on steady state availability of the system is discussed. Optimal means "maximizing the steady state availability."

* Department of Applied Physics, Osaka University, Japan. 


\section{Model}

1. The system consists of two dissimilar redundant units $A_{1}$ and $A_{2}$.

2. There is only one repair facility. The service discipline is first come first served.

3. Initially unit $A_{1}$ is switched on and $A_{2}$ is in warm standby.

4. The failure time of unit $A_{i}(i=1,2)$ while in operation (in standby) is assumed to be exponentially distributed with parameter $\lambda_{i}\left(\lambda_{i}^{*}\right)$.

5. The probability density function of the repair time of the unit $A_{i}(i=1,2)$ when it fails while in operation is $g_{i}(t)=\mu_{i}(t) \exp \left[-\int_{0}^{t} \mu_{i}(v) d v\right]$. The corresponding repair density when the unit fails while in standby is $g_{i}^{*}(t)=\mu_{i}^{*}(t)$ exp $\left[-\int_{0}^{t} \mu_{i}^{*}(v) d v\right]$.

6. As soon as the operating unit fails, the switch-over device operates only when the standby unit is not under repair and it switches the failed unit out and the good unit in. The probability of successful operation of switch-over is $b$ (constant), and its failure probability is $(1-b)$. The repair of the failed switchover device is started immediately before the repair of the failed unit. The general probability density function of the repair time of switching device is $g_{3}(t)=\nu(t) \exp$ $\left[-\int_{0}^{t} \nu(v) d v\right]$.

7. The failure and repair processes are assumed to be independent. The repair is started immediately whenever the server is free and repaired units and switch behave like new after repair.

8. The system fails if an operating unit fails while the other is still under repair, or if the switch-over device fails. When the system is failed, the failure rate of each good unit is zero.

The system can be identified in any one of the state, at any time instant:

$E_{i}$ denotes that $A_{i}$ is in active state and $A_{3-i}$ is in standby state $(i=1,2)$.

$E_{2+i}$ denotes that $A_{i}$ is in active state and $A_{3-i}$ is undergoing repair owing to failure while in active state $(i=1,2)$.

$E_{4+i}$ denotes that $A_{i}$ is in active state and $A_{3-i}$ is undergoing repair owing to failure while in standby state $(i=1,2)$.

$F_{i} \quad$ denotes that $A_{i}$ is undergoing repair with density $g_{i}(t)$ and $A_{3-i}$ is queueing for repair owing to failure while in active state $(i=1,2)$.

$F_{2+i}$ denotes that $A_{i}$ is undergoing repair with density $g_{i}^{*}(t)$ and $A_{3-i}$ is queuing for repair owing to failure while in active state $(i=1,2)$.

$F_{4+i}$ denotes that the switch-over device is undergoing repair and $A_{i}$ and $A_{3-i}$ are queueing for repair owing to failure while in active state and are in standby, respectively, $(i=1,2)$.

By noting the assumption 6, the one-step transition between the state are given by Fig. 1. If we assume that the switch-over device does not fail when a unit is under repair and the failure of the switch-over device is detected after its operation, though the meaning of $b$ is different all the results and equations are the 


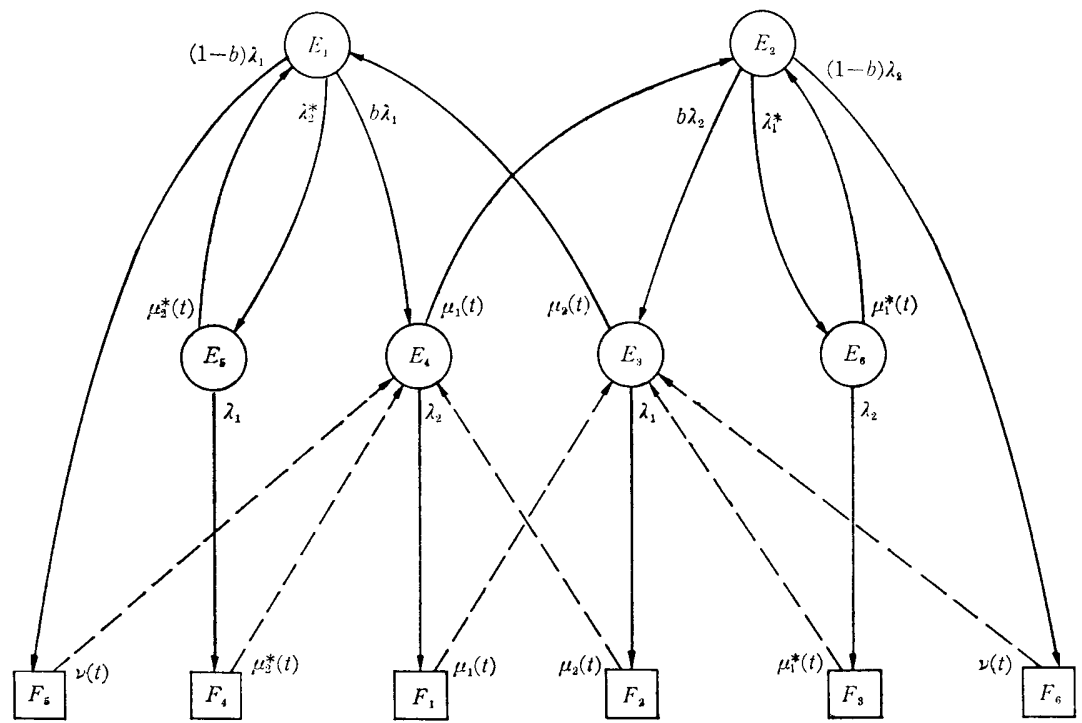

Fig. 1. The state transition diagram.

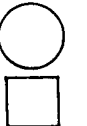

System Operating.

System Failed.

same.

We now introduce the following notations:

$\alpha_{j}, \alpha_{j}^{*}$ first moment of $g_{j}(t)$ and $g_{j}^{*}(t)$

$\hat{g}(s)$ Laplace transform of $g(t)$

$\hat{\alpha}_{j}(s)=s+\lambda_{j}+\lambda_{3-j}^{*}\left[1-g_{3-j}^{*}\left(s+\lambda_{j}\right)\right]$

$\hat{\beta}_{j}(s)=\hat{g}_{j}(s)-\hat{g}_{j}\left(s+\lambda_{3-j}\right)$

$\hat{\beta}_{j}^{*}(s)=\hat{g}_{i}^{*}(s)-\hat{g}_{j}^{*}\left(s+\lambda_{3-j}\right)$

$\hat{\beta}_{3}(s)=b+(1-b) \hat{g}_{3}(s)$

$\varepsilon(t)$ denotes the elapsed repair time of the unit currently in service, if any, at time $t$.

$p_{j}(t)=\operatorname{Pr}\left\{E_{j}(t) \mid E_{1}(0)\right\} \quad(j=1, \cdots, 6)$

$p_{A}(t)=\operatorname{Pr}$ at time $t$, the system is functioning $\left.\mid E_{1}(0)\right\}$

$$
=\sum_{j=1}^{6} p_{j}(t)
$$

$p_{j}(t, x) d x+o(d x)=\operatorname{Pr}\left\{E_{j}(t) \cap[x<\varepsilon(t)<x+d x] \mid E_{1}(0)\right\} \quad(j=3, \cdots, 6)$

$q_{j}(t)=\operatorname{Pr}\left\{F_{j}(t) \mid E_{1}(0)\right\} \quad(j=1, \cdots, 6)$

$q_{D}(t)=\operatorname{Pr}$ at time $t$, the system is in failed state $\left.\mid E_{1}(0)\right\}$

$$
=\sum_{j=1}^{6} q_{j}(t)
$$

$q_{j}(t, x) d x+o(d x)=\operatorname{Pr}\left\{F_{j}(t) \cap[x<\varepsilon(t)<x+d x] \mid E_{1}(0)\right\} \quad(j=1, \cdots, 6)$

$R(t)=\operatorname{Pr}$ the time of faultless operation of the system will be at least $\left.t \mid E_{1}(0)\right\}$ $\mathrm{MTSF}=$ mean tine to system failure 


\section{System Availability}

Viewing the nature of the system, the following set of integro-differential equations can easily be set up (see Fig. 1):

$$
\begin{aligned}
& \left\{\frac{d}{d t}+\lambda_{1}+\lambda_{2}^{*}\right\} p_{1}(t)=\int_{0}^{t} p_{3}(t, x) \mu_{2}(x) d x+\int_{0}^{t} p_{5}(t, x) \mu_{2}^{*}(x) d x \\
& \left\{\frac{d}{d t}+\lambda_{1}^{*}+\lambda_{2}\right\} p_{2}(t)=\int_{0}^{t} p_{4}(t, x) \mu_{1}(x) d x+\int_{0}^{t} p_{6}(t, x) \mu_{1}^{*}(x) d x \\
& \left\{\frac{\partial}{\partial t}+\frac{\partial}{\partial x}+\left[\lambda_{1}+\mu_{2}(x)\right]\right\} p_{3}(t, x)=0 \\
& \left\{\frac{\partial}{\partial t}+\frac{\partial}{\partial x}+\left[\lambda_{2}+\mu_{1}(x)\right]\right\} p_{4}(t, x)=0 \\
& \left\{\frac{\partial}{\partial t}+\frac{\partial}{\partial x}+\left[\lambda_{1}+\mu_{2}^{*}(x)\right]\right\} p_{5}(t, x)=0 \\
& \left\{\frac{\partial}{\partial t}+\frac{\partial}{\partial x}+\left[\lambda_{2}+\mu_{1}^{*}(x)\right]\right\} p_{6}(t, x)=0 \\
& \left\{\frac{\partial}{\partial t}+\frac{\partial}{\partial x}+\mu_{1}(x)\right\} q_{1}(t, x)=\lambda_{2} p_{4}(t, x) \\
& \left\{\frac{\partial}{\partial t}+\frac{\partial}{\partial x}+\mu_{2}(x)\right\} q_{2}(t, x)=\lambda_{1} p_{3}(t, x) \\
& \left\{\frac{\partial}{\partial t}+\frac{\partial}{\partial x}+\mu_{1}^{*}(x)\right\} q_{3}(t, x)=\lambda_{2} p_{6}(t, x) \\
& \left\{\frac{\partial}{\partial t}+\frac{\partial}{\partial x}+\mu_{2}^{*}(x)\right\} q_{4}(t, x)=\lambda_{1} p_{5}(t, x) \\
& \left\{\frac{\partial}{\partial t}+\frac{\partial}{\partial x}+\nu(x)\right\} q_{j}(t, x)=0 \quad(j=5,6)
\end{aligned}
$$

The above equations are to be solved subject to the following boundary and initial conditions ;

$$
\begin{aligned}
& p_{3}(t, 0)=\lambda_{2} b p_{2}(t)+\int_{0}^{t} q_{1}(t, x) \mu_{1}(x) d x+\int_{0}^{t} q_{3}(t, x) \mu_{1}^{*}(x) d x+\int_{0}^{t} q_{6}(t, x) \nu(x) d x \\
& p_{4}(t, 0)=\lambda_{1} b p_{1}(t)+\int_{0}^{t} q_{2}(t, x) \mu_{2}(x) d x+\int_{0}^{t} q_{4}(t, x) \mu_{2}^{*}(x) d x+\int_{0}^{t} q_{5}(t, x) \nu(x) d x \\
& p_{5}(t, 0)=\lambda_{2}^{*} p_{1}(t) \\
& p_{6}(t, 0)=\lambda_{1}^{*} p_{2}(t) \\
& q_{j}(t, 0)=0 \quad(j=1, \cdots, 4) \\
& q_{5}(t, 0)=\lambda_{1}(1-b) p_{1}(t) \\
& q_{6}(t, 0)=\lambda_{2}(1-b) p_{2}(t) \\
& p_{1}(0)=0
\end{aligned}
$$

Taking the Laplace transforms of equations (1)-(6) and (14)--(15) and noting that $p_{j}(t)=\int_{0}^{t} p_{j}(t, x) d x(j=3, \cdots, 4)$, we obtain after some simplification, 


$$
\begin{aligned}
& \hat{p}_{1}(s)=\frac{1+\hat{g}_{2}\left(s+\lambda_{1}\right) \hat{p}_{3}(s, 0)}{\hat{\alpha}_{1}(s)} \\
& \hat{p}_{2}(s)=\frac{\hat{g}_{1}\left(s+\lambda_{2}\right) \hat{p}_{4}(s, 0)}{\hat{\alpha}_{2}(s)} \\
& \hat{p}_{3}(s)=\frac{\left[1-\hat{g}_{2}\left(s+\lambda_{1}\right)\right] \hat{p}_{3}(s, 0)}{s+\lambda_{1}} \\
& \hat{p}_{4}(s)=\frac{\left[1-\hat{g}_{1}\left(s+\lambda_{2}\right)\right] \hat{p}_{4}(s, 0)}{s+\lambda_{2}} \\
& \hat{p}_{5}(s)=\frac{\lambda_{2}^{*}\left[1-\hat{g}_{2}^{*}\left(s+\lambda_{1}\right)\right]\left[1+\hat{g}_{2}\left(s+\lambda_{1}\right) \hat{p}_{3}(s, 0)\right]}{\left(s+\lambda_{1}\right) \hat{\alpha}_{1}(s)}
\end{aligned}
$$

and

$$
\hat{p}_{6}(s)=\frac{\lambda_{1}^{*}\left[1-\hat{g}_{1}^{*}\left(s+\lambda_{2}\right)\right] \hat{g}_{1}\left(s+\lambda_{2}\right) \hat{p}_{4}(s, 0)}{\left(s+\lambda_{2}\right) \hat{\alpha}_{2}(s)}
$$

Substituting the solution of (3)-(6) into (7)-(11) and taking the Laplace transforms, wo obtain

$$
\begin{aligned}
& \hat{q}_{1}(s, x)=\hat{p}_{4}(s, 0)\left\{\exp \left[-s x-\int_{0}^{x} \mu_{1}(z) d z\right]-\exp \left[-\left(s+\lambda_{2}\right) x-\int_{0}^{x} \mu_{1}(z) d z\right]\right\} \\
& \hat{q}_{2}(s, x)=\hat{p}_{3}(s, 0)\left\{\exp \left[-s x-\int_{0}^{x} \mu_{2}(z) d z\right]-\exp \left[-\left(s+\lambda_{1}\right) x-\int_{0}^{x} \mu_{2}(z) d z\right]\right\} \\
& \hat{q}_{3}(s, x)=\hat{p}_{6}(s, 0)\left\{\exp \left[-s x-\int_{0}^{x} \mu_{1}^{*}(z) d z\right]-\exp \left[-\left(s+\lambda_{2}\right) x-\int_{0}^{x} \mu_{1}^{*}(z) d z\right]\right\} \\
& \hat{q}_{4}(s, x)=\hat{p}_{5}(s, 0)\left\{\exp \left[-s x-\int_{0}^{x} \mu_{2}^{*}(z) d z\right]-\exp \left[-\left(s+\lambda_{1}\right) x-\int_{0}^{x} \mu_{2}^{*}(z) d z\right]\right\} \\
& \hat{q}_{5}(s, x)=\lambda_{1}(1-b) \hat{p}_{1}(s) \exp \left[-s x-\int_{0}^{x} \nu(z) d z\right]
\end{aligned}
$$

and

$$
\hat{q}_{6}(s, x)=\lambda_{2}(1-b) \hat{p}_{2}(s) \exp \left[-s x-\int_{0}^{x} \nu(z) d z\right]
$$

Taking the Laplace transforms of (12) and (13) and using (26)-(31) we have

$$
\hat{p}_{3}(s, 0)=\lambda_{2} \hat{p}_{2}(s) \hat{\beta}_{3}(s)+\hat{p}_{4}(s, 0) \hat{\beta}_{1}(s)+\hat{p}_{6}(s, 0) \hat{\beta}_{1}^{*}(s)
$$

and

$$
\hat{p}_{4}(s, 0)=\lambda_{1} \hat{p}_{1}(s) \hat{\beta}_{3}(s)+\hat{p}_{3}(s, 0) \hat{\beta}_{2}(s)+\hat{p}_{5}(s, 0) \hat{\beta_{2}^{*}}(s)
$$

$\hat{p}_{3}(s, 0)$ and $\hat{p}_{4}(s, 0)$ can be obtained explicitly from the Laplace transforms of (14) and (15) and equations (20), (21), (32) and (33):

$$
\begin{aligned}
& \hat{p}_{3}(s, 0)=\frac{\left[\lambda_{1} \hat{\beta}(s)+\lambda_{2}^{*} \hat{\beta}_{2}^{*}(s)\right]\left\{\hat{\alpha}_{2}(s) \hat{\beta}_{1}(s)+\left[\lambda_{2} \hat{\beta}_{3}(s)+\lambda_{1}^{*} \hat{\beta}_{1}^{*}(s)\right] \hat{g}_{1}\left(s+\lambda_{2}\right)\right\}}{\prod_{j=1}^{2} \hat{\alpha}_{j}(s)-\prod_{j=1}^{2}\left\{\hat{\alpha}_{j}(s) \hat{\beta}_{3-j}(s)+\left[\lambda_{j} \hat{\beta}_{3}(s)+\lambda_{3-j}{ }_{-j} \hat{\beta}_{3-j}(s)\right] \hat{g}_{3-j}\left(s+\lambda_{j}\right)\right\}} \\
& \hat{p}_{4}(s, 0)=\frac{\hat{\alpha}_{2}(s)\left[\lambda_{1} \hat{\beta}_{3}(s)+\lambda_{2}^{*} \hat{\beta}_{2}^{*}(s)\right]}{\prod_{j=1}^{2} \hat{\alpha}_{j}(s)-\prod_{j=1}^{2}\left\{\hat{\alpha}_{j}(s) \hat{\beta}_{3-j}(s)+\left[\lambda_{j} \hat{\beta}_{3}(s)+\lambda_{3-j}^{*} \hat{\beta}_{3-j}^{*}(s)\right] \hat{g}_{3-j}\left(s+\lambda_{j}\right)\right\}}
\end{aligned}
$$


The system availability $p_{A}(t)$ and $q_{D}(t)$, the probability that the system is in down state at time $t$, are obtained from (20)-(25). Hence we obtain the following theorem.

THEOREM 3.1 The Laplace transforms of $p_{A}(t)$ and $q_{D}(t)$ are given by

$$
\begin{aligned}
& \hat{p}_{A}(s)=\frac{1}{s+\lambda_{1}}+\frac{\hat{p}_{3}(s, 0)}{s+\lambda_{1}}+\frac{\hat{p}_{4}(s, 0)}{s+\lambda_{2}} \\
& \hat{q}_{D}(s)=\frac{1}{s}-\hat{p}_{A}(s)
\end{aligned}
$$

where $\hat{p}_{3}(s, 0)$ and $\hat{p}_{4}(s, 0)$ are given as (34) and (35), and the mean down time of the system $E[D(t)]$ is given by

$$
E[D(t)]=\int_{0}^{t} q_{D}(t) d t .
$$

By applying Tauberian theorem to (36) we obtain the following theorem.

THEOREM 3.2 The steady state availability of the system $p_{A}(\infty)$ is given by

$$
\begin{aligned}
p_{A}(\infty) & =\lim _{t \rightarrow \infty} p_{A}(t)=\lim _{s \rightarrow 0} s p_{A}(s) \\
& =\frac{\frac{1}{\lambda_{1}}+\frac{1}{\lambda_{2}}}{\sum_{j=1}^{2}\left\{\alpha_{j}+\frac{\hat{g}_{j}\left(\lambda_{3-j}\right)\left[\left(1+\lambda_{j}^{*} \alpha_{j}^{*}\right) \lambda_{3-j}(1-b) \alpha_{3}\right]}{\lambda_{3-j}+\lambda_{j}^{*}\left[1-g_{j}^{*}\left(\lambda_{3-j}\right)\right]}\right\}}
\end{aligned}
$$

The Laplace transform $\hat{R}(s)$ of the reliabiliiy of the system $R(t)$, can be obtained from $\hat{p}(s)$ by making suitable transformations which are equivalent to the assertion that the probability of the system moving from down state to up state is zero. For example, if we substitute $\hat{\beta}_{j}(s)=0, \hat{\beta}_{j}^{*}(s)=0 \quad(j=1,2)$, and $\hat{g}_{3}(s)=0$ in (36), we have the reliability transform of the system. Because from (26)-(31) we have

$$
\begin{aligned}
& Q_{1} \equiv \int_{0}^{\infty} \hat{q}_{1}(s, x) \mu_{1}(x) d x=\hat{p}_{4}(s, 0) \hat{\beta}_{1}(s) \\
& Q_{2} \equiv \int_{0}^{\infty} \hat{q}_{2}(s, x) \mu_{2}(x) d x=\hat{p}_{3}(s, 0) \hat{\beta}_{2}(s) \\
& Q_{3} \equiv \int_{0}^{\infty} \hat{q}_{3}(s, x) \mu_{1}^{*}(x) d x=\hat{p}_{6}(s, 0) \hat{\beta}_{1}^{*}(s) \\
& Q_{4} \equiv \int_{0}^{\infty} \hat{q}_{4}(s, x) \mu_{2}^{*}(x) d x=\hat{p}_{5}(s, 0) \hat{\beta}_{2}^{*}(s) \\
& Q_{5} \equiv \int_{0}^{\infty} \hat{q}_{5}(s, x) \nu(x) d x=\lambda_{1}(1-b) \hat{p}_{1}(s) \hat{g}_{3}(s) \\
& Q_{6} \equiv \int_{0}^{\infty} \hat{q}_{6}(s, x) \nu(x) d x=\lambda_{2}(1-b) \hat{p}_{2}(s) \hat{g}_{3}(s)
\end{aligned}
$$

where $\hat{p}_{j}(s, 0) \neq 0,(j=3, \cdots, 6) \quad \hat{p}_{j}(s) \neq 0, \quad(j=1,2) \quad b \neq 1$, therefore we obtain $\hat{\beta}_{j}(s)=\hat{\beta}_{j}^{*}(s)=0,(j=1,2)$, and $\hat{g}_{3}(s)=0$ from $Q_{j}=0 \quad(j=1, \cdots, 6)$.

For example, $\hat{\beta}_{1}(s)=0$ means that in transition $E_{1} \rightarrow E_{4} \rightarrow F_{1}$, the Laplace-Stieltjes transform of distribution function of the time of stay in $E_{4}$ and $F_{1}$ is zero. Our 
result may be sumarized in the following theorems.

THEOREM 3.3 The $\hat{R}(s)$ of the system is given by

$$
\hat{R}(s)=\frac{1}{s+\lambda_{1}}+\frac{\lambda_{1} b\left[\left(s+\lambda_{2}\right) \lambda_{2} b \hat{g}_{1}\left(s+\lambda_{2}\right)+\left(s+\lambda_{1}\right) \hat{\alpha}_{2}(s)\right]}{\left(s+\lambda_{1}\right)} \frac{\left(s+\lambda_{2}\right)\left[\hat{\alpha}_{1}(s) \hat{\alpha}_{2}(s)-b^{2} \lambda_{1} \lambda_{2} \hat{g}_{1}\left(s+\lambda_{2}\right) \hat{g}_{2}\left(s+\lambda_{1}\right)\right]}{(s)}
$$

THEOREM 3.4 The MTSF of the system is given by

$$
\begin{aligned}
\mathrm{MTSF} & =\lim _{s \rightarrow 0} \hat{R}(s) \\
& =\frac{1}{\lambda_{1}}+\frac{b\left[\lambda_{2}^{2} b \hat{g}_{1}\left(\lambda_{2}\right)+\lambda_{1} \hat{\alpha}_{2}(0)\right]}{\lambda_{2}\left[\hat{\alpha}_{1}(0) \hat{\alpha}_{2}(0)-b \lambda_{1} \lambda_{2} \hat{g}_{1}\left(\lambda_{2}\right) \hat{g}_{2}\left(\lambda_{1}\right)\right]}
\end{aligned}
$$

We give below the availability functions, reliabılity functions and MTSF of some special case.

\section{(i) Cold standby system with dissimilar units}

$$
\begin{aligned}
& \hat{p}_{A}(s)=\frac{1}{s+\lambda_{1}}\left\{1+\frac{\lambda_{1} \hat{\beta}_{3}(s)\left[s+\lambda_{1}+\lambda_{2} \hat{\beta}_{3}(s) \hat{g}_{1}\left(s+\lambda_{2}\right)+\left(s+\lambda_{2}\right) \hat{\beta}_{1}(s)\right]}{\prod_{j=1}^{2}\left(s+\lambda_{j}\right)-\prod_{j=1}^{2}\left[\left(s+\lambda_{j}\right) \hat{\beta}_{3-j}(s)+\lambda_{j} \hat{\beta}_{3}(s) \hat{g}_{3-j}\left(s+\lambda_{j}\right)\right]}\right\} \\
& p_{A}(\infty)=\frac{\lambda_{1}+\lambda_{2}}{\lambda_{1} \lambda_{2}\left(\alpha_{1}+\alpha_{2}\right)+\lambda_{1} \hat{g}_{1}\left(\lambda_{2}\right)\left[1+\lambda_{2}(1-b) \alpha_{3}\right]+\lambda_{2} \hat{g}_{2}\left(\lambda_{1}\right)\left[1+\lambda_{1}(1-b) \alpha_{3}\right]} \\
& \hat{R}(s)=\frac{1}{s+\lambda_{1}}\left\{1+\frac{\lambda_{1} b\left[s+\lambda_{1}+\lambda_{2} b \hat{g}_{1}\left(s+\lambda_{2}\right)\right]}{\left(s+\lambda_{1}\right)\left(s+\lambda_{2}\right)-\lambda_{1} \lambda_{2} b^{2} \hat{g}_{1}\left(s+\lambda_{2}\right) \hat{g}_{2}\left(s+\lambda_{1}\right)}\right\} \\
& \operatorname{MTSF}=\frac{1}{\lambda_{1}}\left\{1+\frac{b\left[\lambda_{1}+\lambda_{2} b \hat{g}_{1}\left(\lambda_{2}\right)\right]}{\lambda_{2}\left[1-b^{2} \hat{g}_{1}\left(\lambda_{2}\right) \hat{g}_{2}\left(\lambda_{1}\right)\right]}\right\}
\end{aligned}
$$

(50) and (51) in $b=1$ agree with the expression of Osaki [3].

(ii) Warm standby system with similar units

$$
\begin{aligned}
& \hat{p}_{A}(s)=\frac{1}{s+\lambda}\left\{1+\frac{\lambda \hat{\beta}_{3}(s)+\lambda^{*} \hat{\beta}^{*}(s)}{\hat{\alpha}(s)[1-\hat{\beta}(s)]-\left[\lambda \hat{\beta}_{3}(s)+\lambda^{*} \beta^{*}(s)\right] \hat{g}(s+\lambda)}\right\} \\
& p_{A}(\infty)=\frac{\lambda+\lambda^{*}\left(1-\hat{g}^{*}(\lambda)\right)}{\lambda\left\{\hat{g}(\lambda)\left[1+\lambda^{*} \alpha^{*}+\lambda(1-b) \alpha_{3}\right]+\left[\lambda+\lambda^{*}\left(1-\hat{g}^{*}(\lambda)\right)\right]\right\}} \\
& \hat{R}(s)=\frac{1}{s+\lambda}\left\{1+\frac{\lambda b}{\hat{\alpha}(s)-\lambda b \hat{g}(s+\lambda)}\right\} \\
& \operatorname{MTSF}=\frac{1}{\lambda}\left\{1+\frac{\lambda b}{\lambda^{*}\left[1-\hat{g}^{*}(\lambda)\right]+\lambda[1-b \hat{g}(\lambda)]}\right\}
\end{aligned}
$$

(52) and (54) in $b=1$ coincide with the expressions obtained by Srinivasan and Gopalan [4].

\section{(iii) Hot standby system with dissimilar units}

We can obtained the results by putting $\lambda_{i}=\lambda_{i}^{*}$ in warm standby system. Especially when $g_{i}(t)=g_{i}^{*}(t) \quad(i=1,2)$, the reliability is given by

$$
\begin{array}{rl}
R(s)=1 & 1 /\left(s+\lambda_{1}\right)+\lambda_{1} b\left\{\left(s+\lambda_{1}+\lambda_{2}\right)\left[s+\lambda_{1}+\left(\lambda_{2} b-\lambda_{1}\right) \hat{g}_{1}\left(s+\lambda_{2}\right)\right]+\lambda_{1} \lambda_{2} \hat{g}_{1}\left(s+\lambda_{2}\right)(b-1)\right\} \\
& /\left\{( s + \lambda _ { 1 } ) ( s + \lambda _ { 2 } ) \cdot \left\{\left(s+\lambda_{1}+\lambda_{2}\right)\left[s+\lambda_{1}+\lambda_{2}-\lambda_{1} \hat{g}_{1}\left(s+\lambda_{2}\right)-\lambda_{2} \hat{g}_{2}\left(s+\lambda_{1}\right)\right]\right.\right. \\
& \left.\left.+\left(1-b^{2}\right) \lambda_{1} \lambda_{2} \hat{g}_{1}\left(s+\lambda_{1}\right) \hat{g}_{2}\left(s+\lambda_{1}\right)\right\}\right\}
\end{array}
$$


and then the MTSF by

$$
\mathrm{MTSF}=\frac{1}{\lambda_{1}}+\frac{b\left\{\left(\lambda_{1}+\lambda_{1}\right)\left[\lambda_{1}+\left(\lambda_{2} b-\lambda_{1}\right) \hat{g}_{1}\left(\lambda_{2}\right)\right]+\lambda_{1} \lambda_{2} \hat{g}_{1}\left(\lambda_{2}\right)(b-1)\right\}}{\lambda_{2}\left\{\left(\lambda_{1}+\lambda_{2}\right)\left[\lambda_{1}\left(1-g_{1}\left(\lambda_{2}\right)\right)+\lambda_{2}\left(1-g_{2}\left(\lambda_{1}\right)\right)\right]+\left(1-b^{2}\right) \lambda_{1} \lambda_{2} \hat{g}_{1}\left(\lambda_{2}\right) \hat{g}_{2}\left(\lambda_{1}\right)\right\}}
$$

(56) and (57) in $b=1$ agree with expressions obtained by Gaver [1].

\section{Comparison of models}

If the repair time distributions and failure rates are given, we can select the optimal model in parallel and standby model by using some special relationships without calculating the availability for each model. Optimal is used in the sense of maximizing the steady state availability. Let $p_{\boldsymbol{A}}^{p}(\infty)$ and $p_{\boldsymbol{A}}^{s}(\infty)$ denote the availability for parallel and standby model respectively. Noting that we obtain the availability for parallel model by putting $\lambda_{j}^{*}=\lambda_{j}, \alpha_{j}=\alpha_{j}^{*}$ and $b=1$ in (39), we have the following theorem from immediate comparison between $p_{\boldsymbol{A}}^{p}(\infty)$ and $p_{\boldsymbol{A}}^{s}(\infty)$.

THEOREM 4.1

$$
\begin{aligned}
p_{A}^{s}(\infty) \leqq p_{A}^{p}(\infty) & \\
\left\langle(1-b) \alpha_{3} \cdot \sum_{j=1}^{2}\left\{-\frac{\lambda_{3-j} \hat{g}_{j}\left(\lambda_{3-j}\right)}{\lambda_{3-j}+\lambda_{j}^{*}\left(1-g_{j}^{*}\left(\lambda_{3-j}\right)\right)}\right\}\right. & \geqq \sum_{j=1}^{2} \hat{g}_{j}\left(\lambda_{3-j}\right)\left\{\frac{1+\lambda_{j} \alpha_{j}}{\lambda_{3-j}+\lambda_{j}\left(1-\hat{g}_{j}\left(\lambda_{3-j}\right)\right)}\right. \\
& \left.-\frac{1+\lambda_{j}^{*} \alpha_{j}^{*}}{\lambda_{3-j}+\lambda_{j}^{*}\left(1-g_{j}^{*}\left(\lambda_{3-j}\right)\right)}\right\}
\end{aligned}
$$

and, for special case we have

i) identical units with warm standby $\left(\lambda_{3-j}=\lambda_{j}=\lambda, \lambda_{j}^{*}=\lambda^{*} \alpha_{j}=\alpha, \alpha_{j}^{*}=\alpha^{*}\right)$;

$$
\begin{aligned}
& p_{A}^{s}(\infty) \leqq p_{A}^{p}(\infty) \\
\Leftrightarrow & (1-b) \lambda \alpha_{3} \geqq \frac{\left[\lambda+\lambda^{*}\left(1-\hat{g}^{*}(\lambda)\right)\right](1+\lambda \alpha)}{\lambda+\lambda(1-\hat{g}(\lambda))}-\left(1+\lambda^{*} \alpha^{*}\right)
\end{aligned}
$$

ii) identical units with cold standby $\left(\lambda_{3-j}=\lambda_{j}=\lambda, \lambda_{j}^{*}=\lambda^{*}=0, \alpha^{*}=0\right.$;

$$
\begin{aligned}
& p_{A}^{s}(\infty) \equiv p_{A}^{p}(\infty) \\
\Longleftrightarrow & (1-b) \lambda \alpha_{3} \geqq \frac{(1+\lambda \alpha)}{2-\hat{g}(\lambda)}-1
\end{aligned}
$$

If the repair time distribution of units are exponential, i.e., $\mu(t)=\mu$, we have

$$
\begin{aligned}
p_{A}^{p}(\infty) & =\frac{1}{1+(1+2 \rho) /\left(2 \rho^{2}\right)} \\
& p_{A}^{s}(\infty)=\frac{1}{1+\rho(1+K) /(1+\rho)} \\
& p_{A}^{s}(\infty) \leqq p_{A}^{p}(\infty) \\
\Leftrightarrow & K \geqq \frac{1}{2}-\frac{1}{2(1+2 \rho)}
\end{aligned}
$$

where $K=(1-b) \mu \alpha_{3}, \rho=\lambda / \mu$

Table 1 shows the numerical examples of the steady state availability (61) and (62) and Fig. 2 shows the numerical results of (63). 
Table 1. Numerical examples for the steady state availability

\begin{tabular}{|c|c|c|c|c|}
\hline \multirow{2}{*}{$=\frac{p_{A}(\infty)}{\rho}$} & \multirow[t]{2}{*}{$p_{A}^{p}(\infty)$} & \multicolumn{3}{|c|}{$p_{A}^{s}(\infty)$} \\
\hline & & 0.01 & 0.1 & 0.3 \\
\hline 0.01 & 0.9998 & 0.9998 & 0.9989 & 0.9969 \\
\hline 0.05 & 0.9955 & 0.9972 & 0.9929 & 0.9836 \\
\hline 0.1 & 0.9836 & 0.9901 & 0.9821 & 0.9649 \\
\hline 0.2 & 0.9459 & 0.9662 & 0.9524 & 0.9231 \\
\hline 0.3 & 0.8989 & 0.9332 & 0.9155 & 0.8784 \\
\hline 0.4 & 0.8491 & 0.8951 & 0.8750 & 0.8333 \\
\hline 0.5 & 0.8000 & 0.8547 & 0.8333 & 0.7895 \\
\hline 0.6 & 0.7534 & 0.8138 & 0.7921 & 0.7477 \\
\hline 0.7 & 0.7101 & 0.7738 & 0.7522 & 0.7083 \\
\hline 0.8 & 0.6701 & 0.7353 & 0.7143 & 0.6716 \\
\hline 0.9 & 0.6335 & 0.7457 & 0.6786 & 0.6376 \\
\hline 1.0 & 0.6000 & 0.6645 & 0.6452 & 0.6061 \\
\hline
\end{tabular}

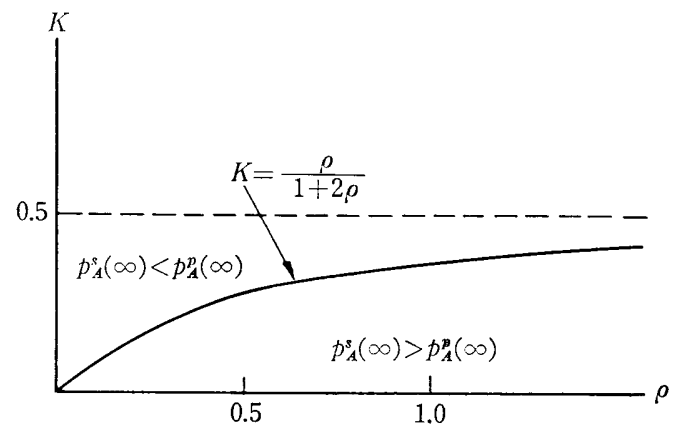

Fig. 2. Comparison of parallel and cold standby model

\section{References}

[1] D.P. Gaver, Failure time for a redundant repairable system of two dissimilar units, IEEE Trans. Reliability, R-13 (1964), 14-22.

[ 2 ] B. V. Gnedenko et al., Mathematical methods of reliability theory, Academic Press, (1969).

[ 3 ] S. OSAKI, Renewal theoretical aspects of two-unit redundant systems, IEEE Trans. Reliability, R-19 (1970), 105-109.

[ 4 ] S.K. SRinivasan and M.N. Gopalan, Probabilistic analysis of a two-unit system with a warm standby and a single repair facility, Oper. Res., 21 (1973), 748-754.

[ 5 ] S. OSAKI, A two-unit standby redundant system with imperfect switchover, IEEE Trans. Reliability, R-21 (1972), 20-24. 